\title{
Tackling Nonatrial Fibrillation Diseases Using Nonvitamin K Antagonist Oral Anticoagulant: by What and for Whom?
}

\author{
Jong S. Kim \\ Department of Neurology, Asan Medical Center, University of Ulsan College of Medicine, Seoul, Korea
}

With an ever-increasing older population, the prevalence of strokes associated with atrial fibrillation (AF) has been on the rise. For stroke neurologists, the use of nonvitamin $\mathrm{K}$ antagonist oral anticoagulants (NOACS) became a treatment option as we can easily prescribe NOACs with less food/drug interactions, no need for regular blood draws or dose adjustment. 1 Based on their convenience of use and trust for efficacy in ischemic stroke prevention, there has been recent increased interest in expanding the use of NOAC in non-AF patients.

In this issue of the Journal of Stroke (JoS), Professor Hong ${ }^{2}$ reviewed the evidence and lack thereof on the use of NOAC in non-AF patients. The review comprehensively covered diseases that can potentially respond to NOAC, such as venous thrombosis, embolic stroke of undetermined source (ESUS), patent foramen ovale (PFO), atherosclerotic diseases, and combined AF and coronary syndromes. Unfortunately, only a few trials that have investigated these issues were successful.

NOACs were not proven to be more effective than aspirin in patients with cerebral venous thrombosis, ESUS, or PFO. Through many trials, two lessons have emerged. First, it appears that only certain, selected patients may benefit from NOAC treatment, such as ESUS patients who are likely to have covert AF. Second, as seen in studies on coronary syndrome and AF, only specific NOACs at certain doses may be effective with acceptable side effects. Therefore, we should consider 'by what and for whom?' when tackling non-AF diseases using NOAC.

Although further studies are undoubtedly needed, solutions may not be readily achieved. Unlike patients with $A F$, patients with ESUS, $\mathrm{PFO}$, or venous thrombosis have a low risk of recurrent stroke and low mortality. This means that trials involving larger numbers of patients are needed to make a statistically reliable conclusion. The rare occurrence of diseases, such as cerebral venous thrombosis, and the presence of competitive drugs, including dual anti-platelets for ath- erosclerotic diseases, will compound difficulties in future studies. Although the review by professor Hong does not give us satisfactory answers in improving our clinical practice, it correctly summarized the current status and future research directions.

Finally, I have two pieces of good news. First, Dr. Edip Gurol from Massachusetts General Hospital, Harvard Medical School has generously accepted to work as an associate editor at JoS. Currently, he is excellently working along with other associate editors, Ji-Hoe, Keun-Sik, and Dong-Eog. Second, the impact factor of JoS has increased from 4.750 to 5.571 in the last year. The current impact factor is higher than all other international journals devoted to clinical stroke, except for Stroke. We are proud that JoS is now placed among the top-ranking international journals. For this remarkable achievement, I am indebted to all the editorial team members for their hard work, contributors who submitted excellent research articles, and those who willingly cited JoS papers in their works.

\section{References}

1. Topcuoglu MA, Liu L, Kim DE, Gurol ME. Updates on prevention of cardioembolic stroke. J Stroke 2018;20:180-196.

2. Hong KS. Non-Vitamin $\mathrm{K}$ antagonist oral anticoagulants in medical conditions at high risk of thromboembolism beyond atrial fibrillation. J Stroke 2019;21:259-275.

Correspondence: Jong S. Kim

Department of Neurology, Asan Medical Center, University of Ulsan College of Medicine, 88 Olympic-ro 43-gil, Songpa-gu, Seoul 05505, Korea

Tel: +82-2-3010-3442

Fax: +82-2-474-4691

E-mail: jongskim@amc.seoul.kr

The author has no financial conflicts of interest. 